\title{
Can insulin-like growth factor 1 (IGF-1), IGF-1 receptor connective tissue growth factor and Ki-67 labelling index have a prognostic role in pulmonary carcinoids?
}

\author{
Georgios A. Kanakis ${ }^{1}$, Lars Grimelius ${ }^{2}$, Dimitrios Papaioannou ${ }^{3}$, Gregory Kaltsas ${ }^{1}$ \\ and Apostolos V. Tsolakis ${ }^{4,5,6}$ \\ ${ }^{1}$ Department of Pathophysiology, Endocrine Unit, University of Athens Medical School, Athens, Greece \\ ${ }^{2}$ Department of Immunology, Genetics and Pathology, Uppsala University, Uppsala, Sweden \\ ${ }^{3}$ Department of Pathology, Hygeia Hospital, Athens, Greece \\ ${ }^{4}$ Department of Oncology and Pathology, Karolinska Institute, Stockholm, Sweden \\ ${ }^{5}$ Cancer Center Karolinska (CCK), Karolinska University Hospital Solna, Stockholm, Sweden \\ ${ }^{6}$ Department of Medical Sciences, Uppsala University, Uppsala, Sweden \\ Correspondence to: Apostolos V. Tsolakis, email: apobtsol@hotmail.com, apostolos.tsolakis@ki.se \\ Keywords: pulmonary carcinoids; IGF-1; CTGF; HIF-1; Ki-67 \\ Received: October 26, $2017 \quad$ Accepted: February 21, $2018 \quad$ Published: April 27, 2018 \\ Copyright: Kanakis et al. This is an open-access article distributed under the terms of the Creative Commons Attribution License \\ 3.0 (CC BY 3.0), which permits unrestricted use, distribution, and reproduction in any medium, provided the original author and \\ source are credited.
}

\section{ABSTRACT}

Introduction: Altered expression of Insulin-like Growth Factor-1 (IGF-1), its receptor (IGF-1R), Connective Tissue Growth Factor (CTGF) and Hypoxia Inducible Factor-1 (HIF-1), has been implicated in tumorigenesis. So far, these factors have not been studied systematically in Pulmonary Carcinoids (PCs).

Aims: To examine IGF-1, IGF-1R, CTGF and HIF-1 expression in PCs, and assess their prognostic value over established factors.

Materials \& Methods: Retrospective study of 121 PCs (104 Typical and 17 Atypical). The expression of growth factors was studied immunohistochemically and tumors were considered positive if immunoreactivity appeared in $>50 \%$ of cells.

Results: All studied parameters were expressed in the majority of tumors (IGF-1, IGF-1R, CTGF and HIF-1, in $78.5 \%, 67 \%, 72 \%$ and $78 \%$, respectively). Their expression tended to be more frequent in TCs and in tumors with Ki-67 $\leq 2 \%$ (significant only for HIF$1 ; 82$ vs. $53 \%$; $p=0.023$ and 83 vs. $63 \%$; $p=0.025$ respectively). CTGF was the only factor correlated with more extensive disease (larger size; presence of lymph node and distant metastases). According to logistic regression analysis, only advanced age, $\mathrm{Ki}-67 \geq 3.4 \%$ and lymph node involvement could predict the development of distant metastases.

Conclusions: IGF-1, IGF-1R, CTGF and HIF-1 are avidly expressed in PCs; however, their presence did not appear to be of statistically significant value over established prognostic factors.

\section{INTRODUCTION}

Pulmonary carcinoids (PCs) are rare tumors of the lungs with neuroendocrine morphology and differentiation. According to the criteria introduced already in the 1990's by Travis et al. [1], they are classified in Typical (TCs) and Atypical Carcinoids (ACs) that exhibit a mostly low and intermediate malignant potential, respectively. However, information regarding the factors involved in the regulation of the biological behavior of PCs is limited. The European Neuroendocrine Tumors Society (ENETS) has suggested a grading system based on the Ki-67 Labelling index (LI) in order to predict the biological behaviour of gastrenteropancreatic neuroendocrine tumors (GEP-NETs) [2]; however, such grading has not been reliably applied in 
Table 1: Summary of patients' demographic and clinicopathological data

\begin{tabular}{|c|c|c|c|c|c|}
\hline & OVERALL & TYPICAL & ATYPICAL & $\chi^{2}(\mathrm{t})$ & $\mathbf{p}$ \\
\hline & $(n=110)$ & $(n=95)$ & $(n=15)$ & & \\
\hline Sex (Female/Male) & $66 / 44$ & $57 / 38$ & $9 / 6$ & 0.000 & 0.616 \\
\hline Age at diagnosis (years \pm SD) & $51.97 \pm 15.66$ & $52.61 \pm 15.07$ & $51.65 \pm 18.54$ & 0.203 & 0.842 \\
\hline Size of primary tumour $(\mathrm{mm} \pm \mathrm{SD})$ & $20.55 \pm 12.21$ & $19.83 \pm 11.42$ & $24.88 \pm 15.94$ & 1.253 & 0.226 \\
\hline $\begin{array}{l}\text { Patients with Localized disease, } \\
\mathrm{T}_{\text {any }} \mathrm{N}_{0} \mathrm{M}_{0}, \mathrm{n}(\%)\end{array}$ & $69(73 \%)$ & $62(76.5 \%)$ & $7(50.0 \%)$ & 4.231 & $0.046^{*}$ \\
\hline $\begin{array}{l}\text { Patients with Lymph Node Metastases, } \\
\mathrm{T}_{\text {any }} \mathrm{N}_{1-{ }_{-3}} \mathrm{M}_{\text {any, }} \mathrm{n}(\%)\end{array}$ & $19(14.7 \%)$ & $15(11.5 \%)$ & $4(21.1 \%)$ & 1.243 & 0.272 \\
\hline $\begin{array}{l}\text { Patients with Distant Metastases, } \\
\mathrm{T}_{\text {any }} \mathrm{N}_{\text {any }} \mathrm{M}_{1,} n(\%)\end{array}$ & $16(16.1 \%)$ & $9(11.8 \%)$ & $7(44 \%)$ & 10.090 & $0.005^{*}$ \\
\hline $\mathrm{Ki}-67 \mathrm{LI} \leq 2 \%$ & $89(73.6 \%)$ & $85(81.7 \%)$ & $4(23.5 \%)$ & \multicolumn{2}{|c|}{$\chi^{2}=25.444$} \\
\hline Ki-67 LI 3-20\% & $32(26.4 \%)$ & $19(18.3 \%)$ & $13(76.5 \%)$ & \multicolumn{2}{|c|}{$\mathrm{p}<0.001^{*}$} \\
\hline Patients with functioning tumors, $n(\%)$ & $9(8.1 \%)$ & $6(6.3 \%)$ & $3(20.0 \%)$ & 3.229 & 0.104 \\
\hline
\end{tabular}

* Correlation is significant (2-tailed).

PCs so far, due to the overlapping distribution of the Ki-67 LI between TCs and ACs [3]. As a result, the initial classification scheme proposed by Travis has been reserved until the most recent WHO 2015 Classification [4]. Moreover, no standard guidelines are currently available on medical therapy for PCs due to their poor response to conventional therapeutic regimens [5]. There is a need to identify new molecular pathways that may serve as biomarkers to better characterize and classify PCs, as well as potential targets for novel molecular therapies.

Several studies have revealed altered expression of Insulin-like Growth Factor-1 (IGF-1) and its receptor (IGF-1R) in different types of human cancers [6-8], particularly in NETs [9-13]. IGF-1 is a factor essential for cell growth, proliferation and differentiation and preclinical studies in NET cell lines have demonstrated that over-expression of IGF-1 and/or IGF-1R, can induce tumor cell proliferation and raise Chromogranin A $(\mathrm{CgA})$ synthesis [14-16]. So far, data regarding the clinical significance of IGF-1 and IGF-1R expression in GEPNETs are inconsistent as in some studies their expression has been associated with a more aggressive behavior and poor prognosis [17], whereas in others no such association could be demonstrated [18]. Only a few studies have investigated the expression of IGF-1 and IGF-1R in PCs without generating robust data $[11,12]$.

Connective Tissue Growth Factor (CTGF) is a member of the cysteine-rich protein 61 , connective tissue growth factor and nephroblastoma overexpressed gene $(\mathrm{CCN})$ proteins family that is present in the extracellular matrix and promotes cellular proliferation and migration [19]. Abnormal amplification of CTGF dependent signals also results in excessive tissue repair, leading to scarring and fibrosis [20]. CTGF expression may be

triggered by serotonin and/or tissue hypoxia that in turn increases Hypoxia Inducible Factor 1 (HIF-1), a nuclear transcription factor, which interacts with the CTGF promoter [21]. CTGF is expressed abundantly in serotonin producing small intestine carcinoids [22] and in gastric NETs [23]. Moreover, a monoclonal antibody directed against CTGF has been demonstrated to attenuate tumour growth and metastasis in a mouse model of pancreatic cancer $[24,25]$. Up to date CTGF expression has not been thoroughly studied in PCs.

The aim of this study is to examine the expression of IGF-1, IGF-1R, CTGF and HIF-1 in PCs by means of immunohistochemistry and assess their prognostic value in comparison to established factors.

\section{RESULTS}

\section{Patient and tumor characteristics}

No significant difference was observed regarding the age at diagnosis and the gender of patients between TCs and ACs (Table 1). The mean tumor size was $20.6 \mathrm{~mm}$ (range 2.0 $-70.0 \mathrm{~mm}$ ) with $93(77 \%)$ tumors exceeding a size of $10 \mathrm{~mm}$ and $21(17 \%)$ of them exceeding $30 \mathrm{~mm}$. ACs were larger than TCs (24.88 vs. $19.83 \mathrm{~mm}$ ), however this difference was not significant $(\mathrm{p}=0.226)$. Patients with ACs presented regional lymph node metastases (LNM), (21\% vs. 11.5\%, $\mathrm{p}=0.272$ ) and/or distant metastases more frequently; however, this difference was significant only for the latter (44\% vs. $12.0 \%, \mathrm{p}=0.005)$. In addition, patients with ACs presented more frequently a functioning syndrome (20\% vs. $6 \%)$, but this difference was also not significant $(p=0.104)$ (Table 1$)$. Urinary 5-HIAA levels were found to be elevated in 4 of 17 patients $(23.5 \%$, mean value $321 \mathrm{mg} / 24 \mathrm{hrs}) ; 2$ of these 
patients harboured ACs and 2 TCs. Regarding biological behaviour, the mean Ki-67 LI was significantly higher in ACs than TCs (6.6 $\pm 4.3 \%$ vs. $1.7 \pm 2.0 \%$; $<<0.001)$. In the majority of ACs (81.7\%) Ki-67 LI was estimated between 3 and $20 \%$, whereas in TCs the majority of tumors $(76.5 \%)$ had a value $\leq 2 \%$. No Grade 3 tumors were identified (the highest Ki-67 LI observed was 12\%).

\section{Controls}

Immunostaining with the antibodies against IGF-1, IGF-1R, CTGF and HIF-1, revealed distinct immunoreactive cells in the corresponding control tissues. At the positive controls IGF-1 and IGF-1R immunoreactivity (IR) was strong. Areas of both moderate and strong intensity were identified at the positive controls for CTGF-and HIF-1. No IR was seen after the replacement of the primary antiserum in question by nonimmune serum, or after the neutralization test, if available.

\section{Immunohistochemistry}

The vast majority ( $>90 \%)$ of the tumor cells in the studied PCs expressed both CgA and Syn. All studied factors were expressed in the majority of tumors (IGF-1, IGF-1R, CTGF, and HIF-1, in 78.5\%, 67\%, 72\% and $78 \%$, of the cases respectively) and the pattern of immunostaining was exclusively cytoplasmic (Figure 1). At the same tumor specimen the intensity of the IR could vary from negative (0) to strong (3) but only the areas of moderate and strong IR were included as positive. Their expression tended to be more frequent in TCs than in ACs, although this was significant only for HIF-1 ( 82 vs. 53\%, $\mathrm{p}=0.023$; Figure 2). In addition, HIF-1 was expressed more frequently among Grade 1 tumors $(83 \%)$ vs. Grade 2 tumors $(63 \%, p=0.025)$, as well as in tumors of small size $(<10 \mathrm{~mm})$, the latter being not significant. On the contrary, CTGF was predominantly expressed among Grade 2 tumors (not significant), tumors measuring $>10 \mathrm{~mm}(79 \%$ vs. $50 \%, \mathrm{p}=0.007$ ), tumors accompanied by regional LNM $(90 \%$ vs. $67 \%$; $\mathrm{p}=0.056)$ as well as by distal metastases $(89 \%$ vs. $65 \% ; \mathrm{p}=0.044)$. The expression of IGF-1 and IGF-1R was not significantly associated with any of the studied parameters, with the exception of IGF-1 being expressed more frequently among non-functioning tumors $(81 \%$ vs. $50 \%$; $p=0.04)$, a trend also observed in the other studied factors (Table 2). Among the 4 patients who had elevated U-5HIAA all their tumors expressed CTGF;
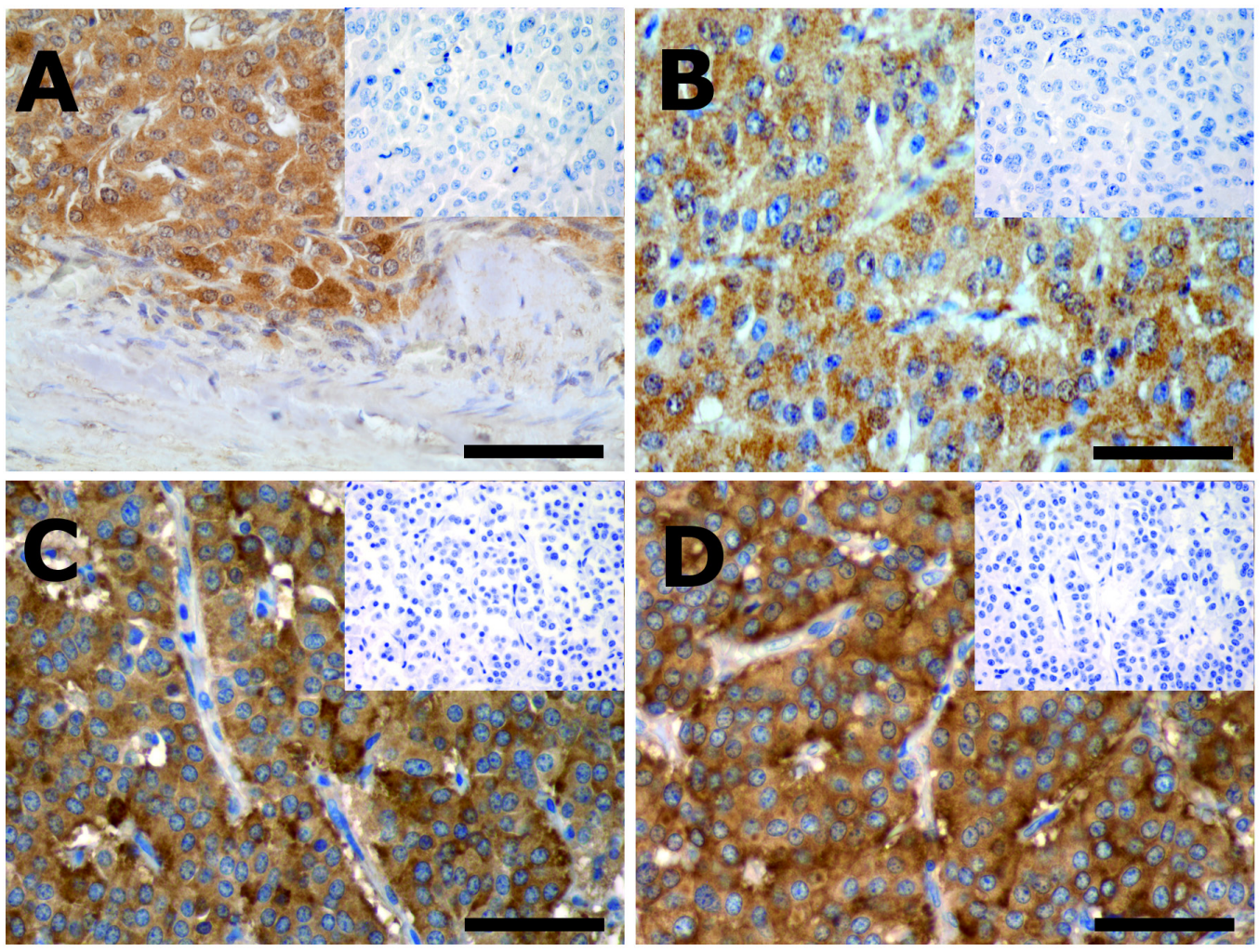

Figure 1: Immunostained sections of a typical (A and B) and an atypical (C and D) pulmonary carcinoid. (A) shows IGF1, (B) IGF-1 receptor, (C) CTGF and (D) HIF-1 immunostained tumor sections. Virtually all tumor cells are immunoreactive and the immunoreactivity is cytoplasmic. The tumor capsule (A) and the fibrovascular stroma (B, C and D) are non-immunoreactive and can be used as internal control. Insets represent microphotographs of the neutralization tests (A, B and C) or replacement of the primary antibody by non-immune serum (D). Scale bars $=100 \mu \mathrm{m}$. 




Figure 2: CTGF, HIF-1, IGF-1 and IGF-1R are expressed in the majority of tumors (A). Their expression tended to be more frequent in TCs, although this was significant only for HIF-1 (B). 
Table 2: CTGF, HIF-1, IGF-1 and IGF-1R expression and co-expression in the 121 studied tumors stratified according to Pathological Diagnosis (PAD), grading, tumor size, disease staging and tumor functionality

\begin{tabular}{|c|c|c|c|c|c|}
\hline & HIF $(+)$ & CTGF(+) & IGF-1(+) & IGF-1R(+) & TOTAL n \\
\hline \multicolumn{6}{|l|}{ PAD } \\
\hline Total & $94(77.7 \%)$ & $87(71.9 \%)$ & $95(78.5 \%)$ & $81(66.9 \%)$ & 121 \\
\hline TC & $85(81.7 \%)$ & $75(72.1 \%)$ & $83(79.8 \%)$ & $72(69.2 \%)$ & 104 \\
\hline $\mathbf{A C}$ & $9(52.9 \%)$ & $12(70.6 \%)$ & $12(70.6 \%)$ & $9(52.9 \%)$ & 17 \\
\hline $\mathbf{x}^{2}(\mathbf{p})$ & $6.986\left(0.023^{*}\right)$ & $0.170(1.000)$ & $0.736(0.523)$ & $1.752(0.265)$ & \\
\hline GRADE & & & & & 121 \\
\hline 1 & $74(83.1 \%)$ & $63(70.8 \%)$ & $68(76.4 \%)$ & $60(67.4 \%)$ & 89 \\
\hline 2 & $20(62.5 \%)$ & $24(75.0 \%)$ & $27(84.4 \%)$ & $21(65.6 \%)$ & 32 \\
\hline $\mathbf{x}^{2}(p)$ & $5.788\left(0.025^{*}\right)$ & $0.206(0.819)$ & $0.886(0.455)$ & $0.034(1.000)$ & \\
\hline $\mathbf{T}$ & & & & & 121 \\
\hline$<=10 \mathrm{~mm}$ & $23(82.1 \%)$ & $14(50.0 \%)$ & $21(75.0 \%)$ & $20(71.4 \%)$ & 28 \\
\hline$>10 \mathrm{~mm}$ & $71(76.3 \%)$ & $73(78.5 \%)$ & $74(79.6 \%)$ & $61(65.6 \%)$ & 93 \\
\hline$x^{2}(p)$ & $0.417(0.612)$ & $8.649\left(0.007^{*}\right)$ & $0.266(0.607)$ & $0.331(0.651)$ & \\
\hline $\mathbf{N}$ & $90^{\dagger}$ & $83^{\dagger}$ & $91^{\dagger}$ & $77^{\dagger}$ & ${ }^{\dagger} 117$ \\
\hline $\mathbf{0}$ & $75(77.3 \%)$ & $65(67.0 \%)$ & $76(78.3 \%)$ & $62(64.0 \%)$ & 97 \\
\hline 1 & $15(75.0 \%)$ & $18(90.0 \%)$ & $15(75.0 \%)$ & $15(75.0 \%)$ & 20 \\
\hline$x^{2}(p)$ & $0.050(0.778)$ & $4.251(0.056)$ & $0.189(0.431)$ & $0.610(0.435)$ & \\
\hline $\mathbf{M}^{\ddagger}$ & 82 & $75^{\ddagger}$ & $83^{*}$ & 69 & $\$ 109$ \\
\hline $\mathbf{0}$ & $71(78.0 \%)$ & $59(64.8 \%)$ & $70(76.9 \%)$ & $60(65.9 \%)$ & 91 \\
\hline 1 & $11(61.1 \%)$ & $16(88.8 \%)$ & $13(72.6 \%)$ & $9(50.0 \%)$ & 18 \\
\hline $\mathbf{x}^{2}(p)$ & $2.306(0.143)$ & $4.050\left(0.044^{*}\right)$ & $0.286(0.555)$ & $1.030(0.312)$ & \\
\hline $\begin{array}{l}\text { Functioning } \\
\text { tumors }\end{array}$ & & & & & 121 \\
\hline No & $88(79.3 \%)$ & $81(72.9 \%)$ & $90(81.1 \%)$ & $75(67.6 \%)$ & 111 \\
\hline Yes & $6(60.0 \%)$ & $6(60.0 \%)$ & $5(50.0 \%)$ & $6(60.0 \%)$ & 10 \\
\hline $\mathbf{x}^{2}(\mathbf{p})$ & $1.967(0.228)$ & $0.7640 .465)$ & $5.253\left(0.037^{*}\right)$ & $0.2320 .433)$ & \\
\hline
\end{tabular}

* Correlation is significant (2-tailed);

${ }^{\dagger}$ data for LNM available for 117 tumors;

¥ data for distant metastases available for 109 tumors.

3 co-expressed IGF-1 with IGF-1R and 2 expressed HIF-1. In three of the patients with increased U-5HIAA, liver metastases were present, associated with high levels of U-5HIAA (mean excretion $640 \mathrm{mmol} / 24 \mathrm{~h}$ ). In the patient without metastases, mean U-5HIAA levels were slightly elevated $(72 \mathrm{mmol} / 24 \mathrm{~h})$

\section{Statistical analysis}

Considering the presence of distant metastases as a surrogate end-point for adverse disease prognosis, correlations with various clinicopathological parameters based on clinical reasoning and on previous publications, were made (Table 3). A significant positive correlation was demonstrated with the age at diagnosis, the pathological classification (AC vs. TC), Ki-67 LI, grade according to ENETS, the presence of regional LNM, and the presence of a secretory syndrome. In all circumstances the correlation strength was low to moderate, with LNM being the most robust predictor of distant metastases. Regarding the immunohistochemically studied factors; only the presence of CTGF was positively correlated to the presence of distant 
metastases; however the significance and strength of the correlation were both marginal. The strength of correlation of the classification scheme currently used by WHO (TC vs. $\mathrm{AC})$ was equivalent to the ENETS Grading system $\left(\mathrm{r}_{\mathrm{s}} \sim 0.3\right)$. Receiver operating characteristic (ROC) curve was used to define the best cut-off point for $\mathrm{Ki}-67 \mathrm{LI}$ that could predict the presence of distant metastases. The area under the ROC curve was $0.670(p=0.023)$ and a Ki-67 LI value of 3.4\% was the optimal cut off with sensitivity $59 \%$ and specificity $81 \%$ (Figure 3).

In order to test the effects of these parameters on the likelihood of developing distant metastases, a logistic regression was performed including gender, age at diagnosis, pathological classification (AC vs. TC), grading according to the suggested $\mathrm{Ki}-67 \mathrm{LI}$ cut off value $(3.4 \%)$, the size of the primary tumor, the presence of regional LNM, the presence of a secretory syndrome, CTGF, HIF, IGF-1 and IGF-1R expression. The logistic regression model was statistically significant $\left(\chi^{2}=27.9, \mathrm{p}\right.$ $<0.001$ ), explained $38.9 \%$ of the variance Nagelkerke $\mathrm{R}^{2}$ ) and correctly classified $90.5 \%$ of cases. However, only age at diagnosis, grading and the presence of regional LNM were significantly associated with an increased likelihood of presenting distant metastases (Table 4).

\section{DISCUSSION}

PCs represent the third most frequent type of NETs after rectal (or gastric NETs according to others) and small intestine NETs and a subset may develop metastatic disease; however there is still a lack of robust data regarding their prognosis and management. In the present study avid expression of, IGF-1, IGF-1R CTGF and HIF1 was documented; however we could not demonstrate a prognostic role, at least superior to established prognostic factors.

Interestingly, the four factors were expressed more frequently in TCs than ACs, though this finding was significant only for HIF-1. In addition, the fact that HIF-1 expression was more frequent in Grade 1 tumors of small size $(<10 \mathrm{~mm})$, without LN or distant metastases, implies an association of HIF-1 expression with less aggressive tumors. On the contrary, CTGF expression was associated with more aggressive disease as it was predominantly expressed on larger tumors $(>10 \mathrm{~mm})$ capable of metastasizing to regional $\mathrm{LN}$ as well as distant organs. Nevertheless, the correlation of CTGF with advanced disease was weak and did not add significant information in the model that predicted the presence of distant metastases. Finally, a similar trend could not be established regarding IGF-1 and IGF-1R expression.

These apparently equivocal findings add more to the controversy that exists regarding the role of these factors in tumorigenesis. For instance, CTGF over-expression has been positively correlated with aggressive behavior of gastric, pancreatic and breast cancer [26-28], whereas it was shown to inhibit metastasis and invasion of colorectal cancer and lung adenocarcinoma [29, 30]. Regarding NETs, CTGF expression is associated with more advanced

\section{Ki-67 LI ROC Curve}

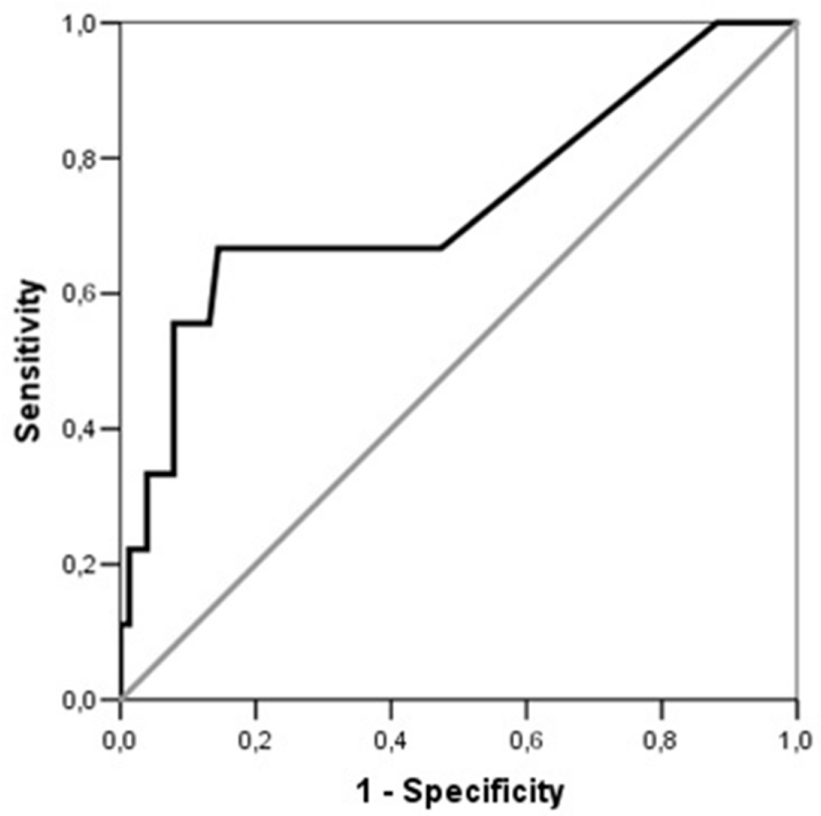

Figure 3: Receiver operating characteristic (ROC) curve illustrating the performance of Ki-67 LI in the prediction of distant metastases in PCs. 
Table 3: Correlations between the presence of metastatic disease and various clinico-pathological parameters in PCs

\begin{tabular}{lccc}
\hline & Spearman's rho & Sig. (2-tailed) & N \\
\hline Gender & 0.043 & 0.696 & 109 \\
Age at diagnosis & 0.263 & $0.005^{*}$ & 109 \\
PAD (TC vs. AC) & 0.304 & $0.001^{*}$ & 109 \\
Ki-67 LI & 0.222 & $0.020^{*}$ & 109 \\
Grade & 0.322 & $0.001^{*}$ & 109 \\
Tumor Size & 0.169 & 0.080 & 109 \\
LNM ${ }^{\text {b }}$ & 0.360 & $<0.001^{*}$ & 109 \\
Functionality $(+)$ & 0.287 & $0.003^{*}$ & 109 \\
CTGF $(+)$ & 0.186 & 0.053 & 109 \\
HIF $(+)$ & -0.145 & 0.131 & 109 \\
CTGF \& HIF $(+)$ & 0.054 & 0.575 & 109 \\
IGF1 $(+)$ & -0.051 & 0.597 & 109 \\
IGF-1R $(+)$ & -0.141 & 0.143 & 109 \\
IGF1 \& IGF1-R $(+)$ & -0.114 & 0.239 & 109 \\
\hline Pathological Diag & & \\
\hline
\end{tabular}

aPathological Diagnosis.

${ }^{\mathrm{b}}$ Lymph Node Metastases.

* Correlation is significant (2-tailed).

Table 4: Summary of logistic regression analysis for variables predicting the presence of distant metastases in patients with PCs

\begin{tabular}{lcccccc}
\hline Variables in the Equation & B & S.E. & Sig. & $\operatorname{Exp}(\mathbf{B})$ & $\mathbf{9 5 , 0 \%}$ C.I.for $\operatorname{EXP(B)}$ & 36,995 \\
\hline LNM & 2,238 & 0,700 & 0,001 & 9,376 & 2,376 & 1,720 \\
GRADE & 1,786 & 0,635 & 0,005 & 5,967 & 1,016 & 1,120 \\
Age at diagnosis & 0,065 & 0,025 & 0,010 & 1,067 & 0,706 \\
Constant & $-6,611$ & 1,674 & 0,000 & 0,001 & & \\
\hline
\end{tabular}

\begin{tabular}{llc}
\hline Variables not in the Equation & Score & Sig. \\
\hline Gender & 0,075 & 0,784 \\
PAD (TC vs. AC) & 2,108 & 0,147 \\
Functionality & 3,421 & 0,064 \\
CTGF(+) & 3,115 & 0,078 \\
HIF-1(+) & 0,323 & 0,570 \\
IGF-1 $(+)$ & 0,788 & 0,375 \\
IGF-1R(+) & 2,953 & 0,086 \\
Size of Primary & 0,000 & 0,997 \\
Overall Statistics & 12,714 & 0,122 \\
\hline
\end{tabular}


disease in ileal and gastric NETs [23, 31]. Similarly, HIF-1 has been recognized in the majority of human cancers as a predictor of poor prognosis [32,33]; however, such data are not available for NETs. Finally a striking discrepancy exists between preclinical and clinical data regarding the IGF-1 / IGF-1R system in NETs, where despite a strong positive association of these factors with tumorigenesis $[34,35]$; relevant anti-IGF-1R therapies have yielded disappointing results [36-38].

The predominant expression of HIF-1 in smaller tumors $(<10 \mathrm{~mm})$ implies that HIF-1 accumulation is an event that occurs early in tumorigenesis, in contrast to CTGF that was mainly expressed in larger tumors $(>10 \mathrm{~mm})$. Consequently, it may be assumed that CTGF expression is an epiphenomenon caused by hypoxia and subsequent HIF-1 accummulation when tumor exceeds a critical size. A similar observation for CTGF has also been made in gastric carcinoids, however at a lower cut off point $(5.0 \mathrm{~mm})$ [23]. On the other hand, CTGF accumulation represents a critical step in tumor development by triggering other pro-angiogenic factors (such as HIF-1 and VEGF) $[39,40]$ and preclinical data have shown that CTGF might serve as a promising potential therapeutic target $[24,25]$.

Recently, much interest has been raised in the treatment of PCs with the mTOR inhibitor everolimus [41]. Preclinical studies suggest that this agent exhibits its antitumor activity through inhibition of IGF-1-dependent pathways [35]. The lack of association of either IGF1 or IGF-1R with other signs of advanced disease, in combination with the non-promising results of anti-IGF-1 / anti-IGF-1R monotherapy implies that this approach alone is not sufficient to block IGF signalling and that the blockade of additional, downstream IGF-1 pathway components is warranted for the favorable results of everolimus to take effect in PCs.

None of the studied factors added substantial information to the prediction of distant metastases, when they were evaluated in a logistic regression model over established prognostic factors, such as advanced age at diagnosis, the presence of regional LNM and the presence of a secretory syndrome (considering such a syndrome as a result of advanced disease status). Furthermore, we could identify a Ki-67 LI cut off value of $3.4 \%$ that was helpful in identifying metastatic from non-metastatic tumors, at least regarding its specificity, and that was also significant in the regression model. This result is in line with others that attempted to apply a grading system for PCs [42]. Reclassification of PCs in two groups according to this cut off (Ki-67 LI <3.4 and $\geq 3.4$ ) correlated better with the WHO classification scheme than the current ENETS Grading system $\left(\mathrm{r}_{\mathrm{s}} 0.542\right.$ vs. $\left.0.492 ; \mathrm{P}=0.001\right)$ and could predict more accurately than both of them the presence of distant metastases $\left(r_{s} 0.322\right.$ vs. 0.317 and 0.304 respectively, $\mathrm{p}=0.001$ )
In conclusion, this study demonstrated that avid expression of CTGF, HIF-1, IGF-1 and its receptor (IGF-1R) exists in PCs. The detection of these factors predominantly in small, well differentiated tumors implies a role at an early stage of tumorigenesis, apart from CTGF, which was expressed in larger more aggressive tumors. However, logistic regression could not yet prove a prognostic role for these factors, implying that they may represent an auto-regulatory mechanism rather than implicate with the biological behavior of the tumors. A limitation of this study is the small number of tumors included, especially ACs, that may compromise its statistical power. However, PCs are rare tumors and the proportion of included TCs to ACs resembles this observed in the general population. On the other hand, the results regarding the use of K-67 LI were promising and a cut off value of $3.4 \%$ could serve as a prognostic factor with sufficient specificity for the likelihood of developing distant metastases.

\section{MATERIALS AND METHODS}

\section{Patients and tumors}

This is a retrospective study of 121 PCs obtained from 110 patients. Some of these tumours have been included in a previous study [43]. Six of the patients harboured multiple pulmonary tumors and two of them tumorlets. In two patients PCs were identified in the context of Multiple Endocrine Neoplasia (MEN) 1. Tumors were classified according to the WHO 2015 criteria [44] in TCs (104 tumors; 86\%) and ACs (17 tumors; 14\%). In 99 patients staging could be assessed according to available clinical data and patients were grouped to those with disease confined in the lung parenchyma $\left(\mathrm{T}_{\text {any }} \mathrm{N}_{0} \mathrm{M}_{0}\right)$, disease involving regional LNM $\left(\mathrm{T}_{\text {any }} \mathrm{N}_{1-3} \mathrm{M}_{\text {any }}\right)$ and advanced disease with distant metastases $\left(\mathrm{T}_{\text {any }} \mathrm{N}_{\text {any }} \mathrm{M}_{1}\right)$. Moreover, the rate of cell proliferation was determined by calculating the $\mathrm{Ki}-67 \mathrm{LI}$ and the tumors were classified in subgroups according to the ENETS Grading classification for GEP NETs (Grade 1: Ki-67 LI $\leq 2 \%$; Grade 2: Ki-67 LI 3-20\%); Grade 3: Ki-67 LI>20\%) [2] (Table 1).

Nine patients presented with functioning tumors (3 with ectopic ACTH secretion, 4 with typical and 2 with atypical carcinoid syndrome). In 17 patients urinary 5-hydroxyl-indolacetic acid (U-5-HIAA) excretion was measured before treatment initiation, (two 24 hour collections using HPLC and calculated as the mean amount; ULN: $60 \mathrm{mmol} / 24 \mathrm{~h}$ ) [45], whereas in one patient with atypical carcinoid syndrome histamine secretion was assessed by measuring urine methylimidazoleacetic acid (UMeImAA), which was found elevated. 


\section{Immunohistochemistry}

The neuroendocrine differentiation of the tumors was confirmed by routine immunostaining with the general neuroendocrine markers $\mathrm{CgA}$ (mouse monoclonal, LK2H10, Boehringer Mannheim, Germany, dilution 1:2000) and synaptophysin (Syn) (rabbit polyclonal, A0010, Dako Cytomation, Glostrup, Denmark, 1:200) using the Dako Autostainer (Dako, Glostrup, Denmark). Furthermore, the sections were also routinely immunostained with the above system with the Ki-67 antibody (mouse monoclonal, Clone MIB-1, M7240, Dako, Denmark, 1:50).

Immunostaining to detect the expression of IGF-1, IGF-1R, CTGF and HIF-1 was applied using the avidinbiotin complex technique (PK-6100, Vectastain ABC, Vector Laboratories Inc., Burlingame, CA, USA) on tissue sections approx. 4 micrometers thick. The sections were incubated with the antibodies overnight at room temperature. Diaminobenzidine was used as chromogen and Meyer's Haematoxylin for nucleus counterstaining. Before immunostaining, the sections were microwave treated for $2 \times 5 \mathrm{~min}$ at $750 \mathrm{~W}$ using Tris buffer saline, $\mathrm{pH}$ 8.0 , as retrieval solution.

The primary and corresponding secondary biotinylated antibodies used were as follows:

- Goat polyclonal IGF-1 (C-20), sc-7144, Santa Cruz, (1: 400); Horse anti-goat $\operatorname{IgG}(\mathrm{H}+\mathrm{L})(\mathrm{BA}-9500$, Vector Laboratories, 1: 100)

- Rabbit polyclonal, IGF-1 Receptor beta (C-20), sc-713 Santa Cruz, (1: 500); Goat anti-rabbit IgG (H + L) (BA 1000, Vector Laboratories, 1: 100)

- Goat polyclonal antibody CTGF L-20, sc-14939, Santa Cruz, (1: 4000); Horse anti-goat IgG (H + L) (BA9500, Vector Laboratories, 1: 100)

- Mouse monoclonal antibody HIF-1 alpha ESEE 122, NB100-131, Novus Biologicals, (1: 3000); Horse antimouse IgG $(\mathrm{H}+\mathrm{L})$ (BA-2000, Vector Laboratories, 1: 100)

\section{Controls}

Tissue from normal liver and kidney were used as positive controls for IGF-1 and IGF-1R respectively, whereas specimens from ileal carcinoid and neuroblastoma served as positive controls for CTGF and HIF-1 respectively. "Negative" immunostaining controls were generated after replacement of the primary antibody by non-immune serum at the same dilution as the primary antibody in question and in the same diluent. In occasional cases, a neutralization test was conducted following a 24hour incubation of the primary antiserum with the relevant antigen (10nmol antigen per ml diluted antibody solution) before application to the sections. The peptides used for the neutralization test were:
- IGF-1 (C-20P), sc-7144P, Santa Cruz

- IGF-1 Receptor beta (C-20P), sc-713P Santa Cruz

- CTGF (L-20P), sc-14939P, Santa Cruz

Neutralization test was not performed for HIF-1 since a relevant peptide was not available.

\section{Intensity score}

The intensity of the immunostaining was divided as 0 for negative, 1 for weak IR, 2 for moderate, and 3 for strong. Tissue areas with only moderate and strong IR were regarded as positive.

\section{Calculation of the relative incidence of immunoreactive cells}

In consecutive immunostained sections, the relative incidence of immunoreactive cells in relation to total tumor area was estimated by light-microscopy at a magnification of X400 using a square grid in one of the oculars. At least five randomly selected areas were examined; in smaller lesions the whole of the neoplastic tissue. Tumors were considered as positive if IR appeared in the majority $(>50 \%)$ of the tumor cells. The Ki-67 LI was calculated by counting the immunoreactive tumor cells at a magnification of X400. To facilitate the counting, a square grid was placed in one of the oculars. At least 2000 tumor cells were counted in the most proliferative tumor areas. The labelling index was expressed as the percentage of Ki-67 immunoreactive cells.

Mitotic activity was estimated in Haematoxylin and Eosin (H\&E) stained tissue sections, analysing 50 High Power Fields, (HPFs) and the mitotic index was expressed as the number of mitoses $/ 10 \mathrm{HPFs}$.

The calculation of Ki-67 and mitoses was performed by one of the authors (LG). The remaining immunohistochemical analysis was done by two investigators (L.G. and A.V.T.) in consensus.

\section{Statistical analysis}

Tumors were stratified according to their size (largest dimension) and two cut off values were selected; $30 \mathrm{~mm}$ and $10 \mathrm{~mm}$. The first cut off point was selected according to the TNM classification system proposed by the American Joint Committee on Cancer, as it is the limit between $\mathrm{T} 1$ and $\mathrm{T} 2$ tumors (AJCC cancer staging manual $7^{\text {th }}$ Ed. 2010), whereas the latter was demonstrated in a previous study to be associated with increased expression of CTGF [23]. For comparisons between nominal and quantitative data Fisher's exact and Student's $t$ test was used respectively, whereas correlations between immunohistochemical findings and clinico-pathological parameters were tested using Spearman's rank correlation coefficient. Receiver Operating Characteristic (ROC) curve was used to assess the performance of the employed 
parameters. A forward stepwise logistic regression was performed to test the effects of different parameters as prognostic factors for the development of distant metastases. The level of statistical significance was set to 0.05 . Calculations were performed using Statistical Package for Social Sciences (SPSS, Inc., Chicago, IL, USA) V.13.0 software.

\section{CONFLICTS OF INTEREST}

Nothing to declare.

\section{Ethics statement}

The research protocol was reviewed and approved by the local research Ethics boards (Uppsala University Hospital, Sweden and University of Athens - Medical School, Greece).

\section{FUNDING}

This work was supported by grants from the Karolinska Institution's Research Foundation and the Hellenic Endocrine Society.

\section{Remarks}

The current original paper was selected as a poster walk oral presentation during the 15th Annual European Neuroendocrine Tumor Society conference held in Barcelona, 7-9 March 2018.

\section{REFERENCES}

1. Travis WD, Rush W, Flieder DB, Falk R, Fleming MV, Gal AA, Koss MN. Survival analysis of 200 pulmonary neuroendocrine tumors with clarification of criteria for atypical carcinoid and its separation from typical carcinoid. Am J Surg Pathol. 1998; 22:934-44. https://doi. org/10.1097/00000478-199808000-00003.

2. Rindi G, Klöppel G, Alhman H, Caplin M, Couvelard A, de Herder WW, Erikssson B, Falchetti A, Falconi M, Komminoth P, Körner M, Lopes JM, McNicol AM, et al, and all other Frascati Consensus Conference participants, and European Neuroendocrine Tumor Society (ENETS). TNM staging of foregut (neuro)endocrine tumors: a consensus proposal including a grading system. Virchows Arch. 2006; 449:395-401. https://doi.org/10.1007/ s00428-006-0250-1.

3. Pelosi G, Rindi G, Travis WD, Papotti M. Ki-67 antigen in lung neuroendocrine tumors: unraveling a role in clinical practice. J Thorac Oncol. 2014; 9:273-84. https://doi. org/10.1097/JTO.0000000000000092.

4. Travis WD, Brambilla E, Burke A, Marx A, Nicholson AG. Who classification of tumours of the lung pleura thymus and heart 2015. Oxford: Oxford University Press (distributor); 2015. 344 p.

5. Caplin ME, Baudin E, Ferolla P, Filosso P, Garcia-Yuste M, Lim E, Oberg K, Pelosi G, Perren A, Rossi RE, Travis WD, and ENETS consensus conference participants. Pulmonary neuroendocrine (carcinoid) tumors: european Neuroendocrine Tumor Society expert consensus and recommendations for best practice for typical and atypical pulmonary carcinoids. Ann Oncol. 2015; 26:1604-20. https://doi.org/10.1093/annonc/mdv041.

6. Maiorano E, Ciampolillo A, Viale G, Maisonneuve P, Ambrosi A, Triggiani V, Marra E, Perlino E. Insulinlike growth factor 1 expression in thyroid tumors. Appl Immunohistochem Mol Morphol. 2000; 8:110-19. https:// doi.org/10.1097/00129039-200006000-00005.

7. Reinmuth N, Fan F, Liu W, Parikh AA, Stoeltzing O, Jung YD, Bucana CD, Radinsky R, Gallick GE, Ellis LM. Impact of insulin-like growth factor receptor-I function on angiogenesis, growth, and metastasis of colon cancer. Lab Invest. 2002; 82:1377-89. https://doi.org/10.1097/01. LAB.0000032411.41603.C2.

8. Cardillo MR, Monti S, Di Silverio F, Gentile V, Sciarra F, Toscano V. Insulin-like growth factor (IGF)-I, IGF-II and IGF type I receptor (IGFR-I) expression in prostatic cancer. Anticancer Res. 2003; 23:3825-35.

9. Wulbrand U, Wied M, Zöfel P, Göke B, Arnold R, Fehmann H. Growth factor receptor expression in human gastroenteropancreatic neuroendocrine tumours. Eur J Clin Invest. 1998; 28:1038-49. https://doi. org/10.1046/j.1365-2362.1998.00397.x.

10. Vitale L, Lenzi L, Huntsman SA, Canaider S, Frabetti F, Casadei R, Facchin F, Carinci P, Zannotti M, Coppola D, Strippoli P. Differential expression of alternatively spliced mRNA forms of the insulin-like growth factor 1 receptor in human neuroendocrine tumors. Oncol Rep. 2006; 15:1249-56.

11. Bowen KA, Silva SR, Johnson JN, Doan HQ, Jackson LN, Gulhati P, Qiu S, Riall TS, Evers BM. An analysis of trends and growth factor receptor expression of GI carcinoid tumors. J Gastrointest Surg. 2009; 13:1773-80. https://doi. org/10.1007/s11605-009-0958-8.

12. Gilbert JA, Adhikari LJ, Lloyd RV, Rubin J, Haluska P, Carboni JM, Gottardis MM, Ames MM. Molecular markers for novel therapies in neuroendocrine (carcinoid) tumors. Endocr Relat Cancer. 2010; 17:623-36. https://doi. org/10.1677/ERC-09-0318.

13. Iida S, Miki Y, Ono K, Akahira J, Suzuki T, Ishida K, Watanabe M, Sasano H. Novel classification based on immunohistochemistry combined with hierarchical clustering analysis in non-functioning neuroendocrine tumor patients. Cancer Sci. 2010; 101:2278-85. https:/doi. org/10.1111/j.1349-7006.2010.01657.x.

14. Nilsson $\mathrm{O}$, Wängberg $\mathrm{B}$, Theodorsson $\mathrm{E}$, Skottner A, Ahlman H. Presence of IGF-I in human midgut carcinoid 
tumours - an autocrine regulator of carcinoid tumour growth? Int J Cancer. 1992; 51:195-203. https://doi. org/10.1002/ijc.2910510206.

15. Von-Wichert G, Jehle PM, Hoeflich A, Wichert V, Koschnick S, Dralle H, Wolf E, Wiedenmann B, Boehm BO, Adler G, Seufferlein T. Insulin-like growth factor-I is an autocrine regulator of chromogranin A secretion and growth in human neuroendocrine tumor cells. Cancer Res. 2000; 60:4573-81.

16. Werner H, Bruchim I. The insulin-like growth factor-I receptor as an oncogene. Arch Physiol Biochem. 2009; 115:58-71. https://doi.org/10.1080/13813450902783106.

17. Furukawa M, Raffeld M, Mateo C, Sakamoto A, Moody TW, Ito T, Venzon DJ, Serrano J, Jensen RT. Increased expression of insulin-like growth factor I and/or its receptor in gastrinomas is associated with low curability, increased growth, and development of metastases. Clin Cancer Res. 2005; 11:3233-42. https://doi.org/10.1158/1078-0432. CCR-04-1915.

18. Wulbrand U, Remmert G, Zöfel P, Wied M, Arnold R, Fehmann HC. mRNA expression patterns of insulin-like growth factor system components in human neuroendocrine tumours. Eur J Clin Invest. 2000; 30:729-39. https://doi. org/10.1046/j.1365-2362.2000.00700.x.

19. Bork P. The modular architecture of a new family of growth regulators related to connective tissue growth factor. FEBS Lett. 1993; 327:125-30. https://doi. org/10.1016/0014-5793(93)80155-N.

20. Shi-Wen X, Leask A, Abraham D. Regulation and function of connective tissue growth factor/CCN2 in tissue repair, scarring and fibrosis. Cytokine Growth Factor Rev. 2008; 19:133-44. https://doi.org/10.1016/j.cytogfr.2008.01.002.

21. Higgins DF, Biju MP, Akai Y, Wutz A, Johnson RS, Haase VH. Hypoxic induction of Ctgf is directly mediated by Hif-1. Am J Physiol Renal Physiol. 2004; 287:F1223-32. https://doi.org/10.1152/ajprenal.00245.2004.

22. Kidd M, Modlin I, Shapiro M, Camp R, Mane S, Usinger W, Murren J. CTGF, intestinal stellate cells and carcinoid fibrogenesis. World J Gastroenterol. 2007; 13:5208-16. https://doi.org/10.3748/wjg.v13.i39.5208.

23. Kaltsas GA, Cunningham JL, Falkmer SE, Grimelius L, Tsolakis AV. Expression of connective tissue growth factor and IGF1 in normal and neoplastic gastrointestinal neuroendocrine cells and their clinico-pathological significance. Endocr Relat Cancer. 2010; 18:61-71. https:// doi.org/10.1677/ERC-10-0026.

24. Aikawa T, Gunn J, Spong SM, Klaus SJ, Korc M. Connective tissue growth factor-specific antibody attenuates tumor growth, metastasis, and angiogenesis in an orthotopic mouse model of pancreatic cancer. Mol Cancer Ther. 2006; 5:1108-16. https://doi.org/10.1158/1535-7163. MCT-05-0516.

25. de Winter P, Leoni P, Abraham D. Connective tissue growth factor: structure-function relationships of a mosaic, multifunctional protein. Growth Factors. 2008; 26:80-91. https://doi.org/10.1080/08977190802025602.

26. Wenger $\mathrm{C}$, Ellenrieder V, Alber B, Lacher U, Menke A, Hameister H, Wilda M, Iwamura T, Beger HG, Adler G, Gress TM. Expression and differential regulation of connective tissue growth factor in pancreatic cancer cells. Oncogene. 1999; 18:1073-80. https://doi.org/10.1038/ sj.onc.1202395.

27. Kang Y, Siegel PM, Shu W, Drobnjak M, Kakonen SM, Cordón-Cardo C, Guise TA, Massagué J. A multigenic program mediating breast cancer metastasis to bone. Cancer Cell. 2003; 3:537-49. https://doi.org/10.1016/ S1535-6108(03)00132-6.

28. Liu L, Li Z, Feng G, You W, Li J. Expression of connective tissue growth factor is in agreement with the expression of VEGF, VEGF-C, -D and associated with shorter survival in gastric cancer. Pathol Int. 2007; 57:712-18. https://doi. org/10.1111/j.1440-1827.2007.02162.x.

29. Lin BR, Chang CC, Che TF, Chen ST, Chen RJ, Yang CY, Jeng YM, Liang JT, Lee PH, Chang KJ, Chau YP, Kuo ML. Connective tissue growth factor inhibits metastasis and acts as an independent prognostic marker in colorectal cancer. Gastroenterology. 2005; 128:9-23. https://doi.org/10.1053/j. gastro.2004.10.007.

30. Chang CC, Yang MH, Lin BR, Chen ST, Pan SH, Hsiao M, Lai TC, Lin SK, Jeng YM, Chu CY, Chen RH, Yang PC, Chin YE, Kuo ML. CCN2 inhibits lung cancer metastasis through promoting DAPK-dependent anoikis and inducing EGFR degradation. Cell Death Differ. 2013; 20:443-55. https://doi.org/10.1038/cdd.2012.136.

31. Svejda B, Kidd M, Giovinazzo F, Eltawil K, Gustafsson BI, Pfragner R, Modlin IM. The 5-HT(2B) receptor plays a key regulatory role in both neuroendocrine tumor cell proliferation and the modulation of the fibroblast component of the neoplastic microenvironment. Cancer. 2010; 116:2902-12. https://doi.org/10.1002/cncr.25049.

32. He J, Hu Y, Hu M, Zhang S, Li B. The relationship between the preoperative plasma level of HIF-1 $\alpha$ and clinic pathological features, prognosis in non-small cell lung cancer. Sci Rep. 2016; 6:20586. https://doi.org/10.1038/ srep20586.

33. Cao S, Yang S, Wu C, Wang Y, Jiang J, Lu Z. Protein expression of hypoxia-inducible factor-1 alpha and hepatocellular carcinoma: a systematic review with metaanalysis. Clin Res Hepatol Gastroenterol. 2014; 38:598603. https://doi.org/10.1016/j.clinre.2014.04.004.

34. Höpfner M, Baradari V, Huether A, Schöfl C, Scherübl H. The insulin-like growth factor receptor 1 is a promising target for novel treatment approaches in neuroendocrine gastrointestinal tumours. Endocr Relat Cancer. 2006; 13:135-49. https://doi.org/10.1677/erc.1.01090.

35. Zatelli MC, Minoia M, Martini C, Tagliati F, Ambrosio MR, Schiavon M, Buratto M, Calabrese F, Gentilin E, Cavallesco G, Berdondini L, Rea F, degli Uberti EC. Everolimus as a 
new potential antiproliferative agent in aggressive human bronchial carcinoids. Endocr Relat Cancer. 2010; 17:719 29. https://doi.org/10.1677/ERC-10-0097.

36. Reidy-Lagunes DL, Vakiani E, Segal MF, Hollywood EM, Tang LH, Solit DB, Pietanza MC, Capanu M, Saltz LB. A phase 2 study of the insulin-like growth factor-1 receptor inhibitor MK-0646 in patients with metastatic, well-differentiated neuroendocrine tumors. Cancer. 2012; 118:4795-800. https://doi.org/10.1002/cncr.27459.

37. Strosberg JR, Chan JA, Ryan DP, Meyerhardt JA, Fuchs CS, Abrams T, Regan E, Brady R, Weber J, Campos T, Kvols LK, Kulke MH. A multi-institutional, phase II open-label study of ganitumab (AMG 479) in advanced carcinoid and pancreatic neuroendocrine tumors. Endocr Relat Cancer. 2013; 20:383-90. https://doi.org/10.1530/ERC-12-0390.

38. Dasari A, Phan A, Gupta S, Rashid A, Yeung SC, Hess K, Chen H, Tarco E, Chen H, Wei C, Anh-Do K, Halperin D, Meric-Bernstam F, Yao J. Phase I study of the anti-IGF1R antibody cixutumumab with everolimus and octreotide in advanced well-differentiated neuroendocrine tumors. Endocr Relat Cancer. 2015; 22:431-41. https://doi. org/10.1530/ERC-15-0002.

39. Chang CC, Lin MT, Lin BR, Jeng YM, Chen ST, Chu CY, Chen RJ, Chang KJ, Yang PC, Kuo ML. Effect of connective tissue growth factor on hypoxia-inducible factor 1alpha degradation and tumor angiogenesis. J Natl Cancer Inst. 2006; 98:984-95. https://doi.org/10.1093/jnci/djj242.

40. Smith EK, Price DK, Figg WD. Piecing together the HIF-1 puzzle: the role of the CTGF as a molecular mechanism of HIF-1 regulation. Cancer Biol Ther. 2006; 5:1443-44. https://doi.org/10.4161/cbt.5.11.3462.

41. Yao JC, Fazio N, Singh S, Buzzoni R, Carnaghi C, Wolin E, Tomasek J, Raderer M, Lahner H, Voi M, Pacaud LB, Rouyrre N, Sachs C, et al, and RAD001 in Advanced Neuroendocrine Tumours, Fourth Trial (RADIANT-4) Study Group. Everolimus for the treatment of advanced, non-functional neuroendocrine tumours of the lung or gastrointestinal tract (RADIANT-4): a randomised, placebocontrolled, phase 3 study. Lancet. 2016; 387:968-77. https://doi.org/10.1016/S0140-6736(15)00817-X.

42. Rindi G, Klersy C, Inzani F, Fellegara G, Ampollini L, Ardizzoni A, Campanini N, Carbognani P, De Pas TM, Galetta D, Granone PL, Righi L, Rusca M, et al. Grading the neuroendocrine tumors of the lung: an evidence-based proposal. Endocr Relat Cancer. 2013; 21:1-16. https://doi. org/10.1530/ERC-13-0246.

43. Kanakis G, Grimelius L, Spathis A, Tringidou R, Rassidakis GZ, Öberg K, Kaltsas G, Tsolakis AV. Expression of Somatostatin Receptors 1-5 and Dopamine Receptor 2 in Lung Carcinoids: Implications for a Therapeutic Role. Neuroendocrinology. 2015; 101:211-22. https://doi. org/10.1159/000381061.

44. Travis WA. Pathology and genetics of tumours of the lung, pleura, thymus and heart. Oxford: Oxford University Press (distributor); 2004. 59-62.

45. Wahlund KG, Edlén B. Simple and rapid determination of 5-hydroxyindole-3-acetic acid in urine by direct injection on a liquid chromatographic column. Clin Chim Acta. 1981; 110:71-76. https://doi.org/10.1016/0009-8981(81)90302-8. 